http://doi.org/10.48195/sepe2021-126

\title{
MECANISMOS NEUROINFLAMATÓRIOS NO COVID-19
}

\section{Rafaela Magalhães Manot Sarrat Lôbo 1 Alciene Pereira da Silva1 Carina Rodrigues Boeck 2 Pedro Roosevelt Torres Romão2}

\section{Resumo:}

Os sobreviventes de COVID-19 apresentam sintomas decorrentes de processos inflamatórios de longo prazo, relacionados aos sintomas como fadiga, dificuldades para dormir, depressão e ansiedade, o que denota a necessidade de conhecer a relação do mecanismo patológico que desencadeia sinais e sintomas neurológicos. $O$ objetivo deste trabalho é desenvolver uma revisão narrativa tendo como pergunta de pesquisa: Quais as evidencias sobre os mecanismos neuroinflamatórias são apontadas como importantes preditores no sintoma a longo prazo de COVID? Tratase de uma revisão bibliográfica elaborada com artigos publicados no site Pubmed, publicados no ano de 2021. Os vinte estudos analisados permitiram conhecer sinteticamente pesquisas científicas atuais que indicam a associação entre os mecanismos inflamatórios e a COVID-19, os pesquisadores apntam sobretudo para a necessidade de entendimento das manifestações neurológicas para melhor qualidade do tratamento da doença.

Palavras-chave: neurologia; COVID-19; inflamação

Eixo Temático: Atenção Integral e Promoção à Saúde (AIPS).

\section{INTRODUÇÃO}

O Corona Virus Disease 2019 (COVID-19) começou a ser notificado em 2020 na China. No contexto brasileiro em fevereiro do mesmo ano também já se notificou o primeiro caso. Porém desde o 2019 mundo inteiro já tinha esta doença como pauta 
de alerta de incertezas no cenário público do setor saúde (DANTAS et al., 2021). Com o avanço da pandemia, verifica-se também a necessidade de conhecer como se apresenta o mecanismo da forma crônica com inflamações sistêmica, e assim foi se identificando sequelas no campo neurológico dos sobreviventes. Junto a doença se desenvolveram manifestações neurológicas e inflamatórias relatadas no processo longo e crônico decorrentes da doença (SOLOMON, 2021).

Diante deste cenário, os cientistas identificaram a necessidade de uma associação entre fatores imunológicos via citocinas inflamatórias, como a interleucina6 (IL-6), e em particular, como este mecanismo poderia sustentar os sintomas de COVID-19 a longo período. A teoria está sendo apoiada por várias linhas de pesquisa, incluindo estudos de coorte de base populacional e estudos de randomização mendeliana genética, sugerindo que a inflamação está associada à desfechos como fadiga e dificuldades para dormir, e que a IL-6 pode representar um possível fator causal para esses sintomas (KAPPELMANN; DANTZER; KHANDAKER, 2021).

A ativação do sistema imune inato e adaptativo em resposta ao SARS-CoV-2 altera o fenótipo e frequencia e/ou função de células inatas e células $\mathrm{T}$ auxiliares, incluindo Th1, Th17 e T reguladoras Treg), podendo afetar mecanismos neurais do aprendizado e os processos emocionais, e levar a um ciclo vicioso de inflamação e disfunção mitocondrial que amplifica o processo neuroinflamatório (HUANG, et al, 2021).

Uma revisão de literatura vem a ser importante na medida em que pode agrupar informações produzidas cientificamente no último ano. Devido a pandemia a produção científica está sendo acelerada e pesquisadores buscam entender os processos neurológicos presentes no mecanismo do COVID-19 grave e crônico. Este trabalho busca sintetizar como o processo inflamatório persistente contribui para a fadiga de longo prazo, gerando patologias neurológicas e diminuindo a qualidade de vida da população que busca a melhor terapêutica no pós-COVID-19.

\section{METODOLOGIA}


Trata-se de uma revisão narrativa onde a busca de informações se deu no site Pubmed através das palavras chaves "neurological" and "inflammation" and "mechanism" no mês de setembro com publicações originais e revisões sistemáticas do ano de 2021. Além do ano, foi utilizado como critério de inclusão os textos completos de acesso livre.

\section{RESULTADOS E DISCUSSÃO}

As consequências do COVID-19 para a saúde a longo prazo permanecem necessitando de investigações científicas, visto que as manifestações neurológicas ainda não estão esclarecidas (HUANG et al., 2021). Porém, é unânime entre estudiosos que pacientes infectados com o vírus SarsCov-2 apresentam uma sintomatologia altamente variável de disfunções do sistema nervoso central (SNC) (RANGANATHAN et al., 2021).

Neste sentido, com objetivo de entender os mecanismos associados ao envolvimento do SNC na COVID-19, Espíndola et al.,(2021) concluiram em que, mesmo aqueles pacientes que apresentaram condições clínicas similares, tiveram indicação de uma possível contribuição da replicação viral no desencadeamento da infiltração do SNC por células imunes e a subsequente inflamação promovendo lesão neuronal. Como a inflamação ja aconetece antes o que pode ocorrer é uma amplificação da resposta inflamatoria no cerebro ou inflamação.

Huang et al., (2021) ao descrever o estado clínico de pessoas sobreviventes da COVID-19 após seis meses de alta e, analisando, suas consequencias com persistencia e até surgimento de novos sintomas relatam que as pessoas apresentavam principalmente fadiga ou fraqueza muscular, dificuldades para dormir e ansiedade ou depressão. Sendo os pacientes que estiveram mais gravemente doentes durante a internação hospitalar, tiveram capacidade de difusão pulmonar com maior gravidade e manifestações anormais de imagem do tórax, e são portanto, a principal população-alvo para intervenção de recuperação a longo prazo.

Ao avaliar a associação da doença COVID com manifestações neurooftalmológicas, Gold ; Galetta (2021) descreveram disfunções do nervo óptico, 
anormalidades do movimento ocular e defeitos do campo visual, sendo, segundo os autores, sintomas e sinais importantes que podem ser o resultado de uma variedade de mecanismos fisiopatológicos ao longo do curso da doença aguda até a fase crônica de recuperação. No campo da otorrinolaringologia, Saussez; Lechien; Hopkins (2021) descrevem a necessidade de continuar as investigações sobre a anosmia, visto que, desde o início da pandemia, muitos médicos e profissionais da saúde em geral, observaram um número sem precedentes de pacientes com perda do olfato. Este sintoma, inclusive, era no inicio da pandemia o primeiro ou até mesmo o único sintoma da infecção por COVID-19, levando a orientações de auto-isolamento nos pacientes afetados.

Ao estudar a morte celular programada e mais especificamente, o efeito da necrostatina-1 (Nec-1) como inibidor da necroptose direcionado ao RIP1, Cao; Mu (2021) apontam para o papel essencial da necroptose em várias doenças, incluindo doenças inflamatórias como a doença provocada pelo o vírus SarsCov-2. Os autores ressaltam que o papel da $\mathrm{Nec}-1$ não tem recebido muita atenção dentro da comunidade científica, mas que ela, por ser capaz de inibir a via de sinalização da necroptose, pode ser aplicada em vários tipos de doenças para aliviar 0 desenvolvimento, melhorar o prognóstico e de proteger contra as complicações.

Perrin et al., (2021) ao estudar a respeito ao papel da síndrome de liberação de citocinas (SRC) no COVID-19 grave destacam que as manifestações neurológicas podem afetar adversamente os resultados clínicos, pois a inflamação e a uremia são fatores de risco para complicações neurológicas como as da encefalopatia relacionada às células $\mathrm{T}$ do receptor do antígeno quimérico. Thepmankorn et al., (2021) aborda neste sentido, que o COVID-19 em pacientes graves pode desencadear um estado hiperinflamatório caracterizado por níveis elevados de citocinas conhecidos como hipercitocinemia ou 'tempestade de citocinas'. Eles ressaltam ainda que, embora COVID-19 seja conhecida por ser uma doença principalmente respiratória, complicações neurológicas que afetam os sistemas nervosos central e periférico também devem ser consideradas ao se descrever a história natural da doença (BOLAY et al., 2021). 
Liu et al . (2021) buscam em seus estudos sobre COVID-19 entender a partir da associação de identificação em pacientes variantes genéticas em envolvidas no risco e prognóstico de COVID-19 e a sua interpretação biológica. Porém, apesar de permanecer sem resposta viável, os resultados fornecem pistas importantes para uma melhor compreensão dos mecanismos de hipercitocinemia e tromboembolismo venoso sendo consequentemente potenciais alvos terapêuticos para o tratamento eficaz de COVID-19.

Alquisiras-Burgos et al. (2021) ao buscar contribuir para a compreensão das condições neurológicas e sua associação com a integridade da barreira hematoencefálica, encorajam o diagnóstico de COVID-19 a partir da presença de aumento no número de citocinas, pois segundo os autores, isto facilitaria os caminhos para o tratamento no sentido que, as complicações neurológicas associadas ao dano à barreira são induzidas pela resposta inflamatória. Os autores discutem inclusive, que esta infecção pode afetar a função cerebral e exacerbar sintomas neurodegenerativos em pacientes, e propõe que as rotas potenciais de neuroinvasão sejam fatores determinantes no mecanismo da doença.

Ao observar que existem notificações regulares de infecções extrapulmonares e manifestações relacionadas com a pandemia de COVID-19 em curso, Kumar et al (2021) abordam que a doença provocada pelo vírus SarsCov-2 são potencialmente neurotrópicos, o que torna o tecido neural vulnerável à infecção, especialmente em idosos ou em indivíduos com comorbidades neurológicas. As queixas de ageusia, anosmia, mialgia e cefaleia, bem como os relatos de doenças como acidente vascular cerebral, encefalopatia, convulsão e encefalite e a perda de consciência em pacientes com COVID-19 confirma o aspecto neuropatofisiológico da doença.

Garget et al (2021) apresentam que existem uma variedade de anormalidades de neuroimagem em COVID-19 e descreve diversos padrões de imagem em pacientes com complicações neurológicas associadas a doença. Indicam em seu estudo de neuroimagem, hiperintensidade da substância branca eriventricular, microssangre, infartos arteriais e venosos e hemorragias como anormalidades distintas em pacientes. 
A interdependência de órgãos vitais pode agravar os aspectos fisiopatológicos de COVID-19 bem como a indução de uma hipercitocinemia na circulação sistêmica pode desencadear uma cascata neuroinflamatória, que pode subsequentemente comprometer a barreira hematoencefálica ativando receptores transmitidos por micróglia e astrócitos, levando a danos no tecido neuronal (KUMAR et al, 2021; ALQUISIRAS-BURGOS et al., 2021).

Ao avaliar a infecção por SARS-CoV-2 por longos períodos em adolescentes e crianças verifica-se a presença de distúrbios psiquiátricos e cognitivos (SINGH et al., 2021). Segundo os autores, o vírus permanece latente no SNC levando a complicações neurológicas. A ampla expressão da enzima conversora de angiotesina (ACE2) no cérebro aumenta a preocupação com seu envolvimento na infecção (SULLIVAN; FISCHER, 2021).

Jesuthasanet et al., (2021) tentam abordar a evolução dos mecanismos celulares neurológicos de longo prazo e dão ênfase às apresentações cerebrovasculares, desmielinizantes e encefalíticas enfatizando que, vários mecanismos, principalmente o envolvimento da hipercitocinemia a longo prazo geram impacto do vírus na cognição, função autonômica e bem-estar mental, representando uma sobre carga para os serviços de saúde.

A doença de Alzheimer surgiu como uma comorbidade da COVID-19 (XIA; WANG; ZHENG, 2021). A mortalidade por COVID-19 é elevada na doença de Alzheimer devido a múltiplas alterações patológicas dos pacientes como, por exemplo, a expressão excessiva do receptor viral da enzima conversora da angiotensina II e as moléculas pró-inflamatórias. O inverso também já foi relatado, ou seja, com a cronificação da doença coronavírus 2019 causada pela síndrome respiratória aguda grave coronavírus-2, também foi relatado vários sintomas neurológicos, incluindo prejuízo cognitivo que pode resultar em doença de Alzheimer, e isso provavelmente por meio da invasão do vírus no sistema nervoso central induzindo o delírio e a demência na síndrome pós-COVID-19.

Gholami et al., (2021) chamam também a atenção para o fato que a coronavírus, como a Síndrome Respiratória Aguda Grave (SARS), a Síndrome do Coronavírus Respiratório do Oriente Médio (MERS) e COVID-19, podem danificar os 
nervos diretamente por meio de disfunções endovasculares, afetando assim as estruturas nervosas e as conexões sinápticas. A linhagem coronavírus também podem induzir a degeneração das células neurais indiretamente por meio da disfunção mitocondrial induzindo estresse oxidativo, inflamação e apoptose. Assim, existe a possibilidade do vírus desencadear a longo prazo distúrbios neurológicos induzindo disfunção neurovascular que afeta as estruturas nervosas e as conexões sinápticas e provocando inflamação, estresse oxidativo e apoptose e que, embora alguns desses mecanismos sejam semelhantes a outros vírus de RNA, os mecanismos neurotóxicos são desconhecidos e precisam de estudos clínicos e experimentais detalhados.

Os estudiosos dos mecanismos neurológicos das doenças causadas por toda linhagem do cornonavírus e sua relação com o processo inflamatorio resultante da doença expõe, no geral, que citocinas pró-inflamatórias, poderiam explicar os sintomas neurológicos de longo prazo após a infecção, além de apontar caminhos para o diagnóstico mais acertivo e o tratamento com maior respostas positivas de sobrevida com qualidade.

\section{CONCLUSÃO}

O desenvolvimento deste trabalho permitiu conhecer sinteticamente alguns caminhos apontados por estudiosos da associação de mecanismos inflamatórios e a COVID-19. Os autores apontam para a necessidade do entendimento de mecanismos patogênicos da doença e em particular, a identificação da presença de hipercitocinemia em pacientes com manifestações neurológicas que facilitará os caminhos para o tratamento. Investigações futuras e pesquisas aprofundadas sobre os níveis de citocinas em pacientes com COVID-19 com sintomas neurológicos, bem como a eficácia dos tratamentos de citoquinas direcionados à tempestade, serão essenciais na elucidação dos mecanismos patogênicos e tratamentos eficazes de COVID-19.

O livre acesso aos textos completos em uma grande base de dados com publicações atuais e de contextos mundiais, possibilitou sistematizar esta pesquisa 
com o desenvolvimento de um compilado importante sobre a relação dos agravos neurológicos a longo prazo e a cronificação do COVID-19.

\section{REFERÊNCIAS}

ALQUISIRAS-BURGOS I, PERALTA-ARRIETA I, ALONSO-PALOMARES LA, ZACAPALA-GÓMEZ AE, SALMERÓN-BÁRCENAS EG, AGUILERA P. Neurological Complications Associated with the Blood-Brain Barrier Damage Induced by the Inflammatory Response During SARS- CoV-2 Infection. Mol Neurobiol. 2021 Feb;58(2):520-535. doi: 10.1007/s12035-020- 02134-7. Epub 2020 Sep 25. PMID: 32978729; PMCID: PMC7518400. Acesso: 15.09.2021.

BOLAY H, KARADAS Ö, OZTÜRK B, SONKAYA R, TASDELEN B, BULUT TDS, GÜLBAHAR Ö, ÖZGE A, BAYKAN B. HMGB1, NLRP3, IL-6 and ACE2 levels are elevated in COVID-19 with headache: a window to the infection-related headache mechanism. J Headache Pain. 2021 Aug 12;22(1):94. doi: 10.1186/s10194-02101306-7. PMID: 34384355; PMCID: PMC8358545. Acesso: 15.09.2021

CAO L, MU W. Necrostatin-1 and necroptosis inhibition: Pathophysiology and therapeutic implications. Pharmacol Res. 2021 Jan;163:105297. doi: 10.1016/j.phrs.2020.105297. Epub 2020 Nov 9. PMID: 33181319; PMCID: PMC7962892. Acesso: 15.09.2021

DANTAS, ESO. Saúde mental dos profissionais de saúde no Brasil no contexto da pandemia por Covid-19. Interface - Comunicação, Saúde, Educação [online]. 2021, v. 25, suppl 1 [Acessado 13 Setembro 2021] , e200203. Disponível em: <https://doi.org/10.1590/Interface.200203>. Epub 08 Jan 2021. ISSN 1807-5762. https://doi.org/10.1590/Interface.200203.Acesso: 13.09. 2021. 
ESPÍNDOLA OM, BRANDÃO CO, GOMES YCP, SIQUEIRA M, SOARES CN, LIMA MASD, LEITE ACCB, TOREZANI G, ARAUJO AQC, SILVA MTT. Cerebrospinal fluid findings in neurological diseases associated with COVID-19 and insights into mechanisms of disease development. Int J Infect Dis. 2021 Jan;102:155-162. doi: 10.1016/j.jij.2020.10.044. Epub 2020 Oct 28. PMID: 33127503; PMCID: PMC7591319. Acesso: 15.09.2021.

GARG RK, PALIWAL VK, MALHOTRA HS, SHARMA PK. Neuroimaging Patterns in Patients with COVID-19-Associated Neurological Complications: A Review. Neurol India. 2021 Mar-Apr;69(2):260-271. doi: 10.4103/0028-3886.314531. PMID: 33904434.Acesso: 13.09,2021.

GHOLAMI M, SAFARI S, ULLOA L, Motaghinejad M. Neuropathies and neurological dysfunction induced by coronaviruses. J Neurovirol. 2021 Jun;27(3):380-396. doi: 10.1007/s13365-021-00977-x. Epub 2021 May 13. PMID: 33983506; PMCID: PMC8117458. Acesso: 13.09,2021.

GOLD DM, GALETTA SL. Neuro-ophthalmologic complications of coronavirus disease 2019 (COVID-19). Neurosci Lett. 2021 Jan 18;742:135531. doi: 10.1016/j.neulet.2020.135531. Epub 2020 Nov 25. PMID: 33248158; PMCID: PMC7687583. Acesso: 13.09,2021.

HUANG C., HUANG L., WANG, YEMING, LI X., REN L., GU X., KANG L., GUO L., LIU M., ZHOU X., LUO J., HUANG Z., TU S., ZHAO Y., CHEN L., LI XU, D., YANPING, LI C., LI PENG, L., YONG, XIE W., CUI D., SHANG L., FAN G., XU J., WANG G., WANG YING, ZHONG J., WANG C., WANG J., ZHANG D., CAO B. Vol. 397. 2021. 6-month consequences of COVID-19 in patients discharged from hospital: a cohort study; pp. 220-232. Lancet. Acesso: 13.09,2021.

JESUTHASAN A, MASSEY F, MANJI H, ZANDI MS, WIETHOFF S. Emerging potential mechanisms and predispositions to the neurological manifestations of 
COVID-19. J Neurol Sci. 2021 Sep 15;428:117608. doi: 10.1016/j.jns.2021.117608.

Epub 2021. Aug 4. PMID: 34391037; PMCID: PMC8332920. Acesso: 13.09,2021.

KAPPELMANN N, DANTZER R, KHANDAKER GM. Interleukin-6 as potential mediator of long-term neuropsychiatric symptoms of COVID-19.

Psychoneuroendocrinology. 2021 Sep;131:105295. doi:

10.1016/j.psyneuen.2021.105295. Epub 2021 Jun 3.

PMID: 34119855; PMCID: PMC8172271. Acesso: 13.09,2021.

KUMAR D, JAHAN S, KHAN A, SIDDIQUI AJ, REDHU NS, WAHAJUDDIN, KHAN J, BANWAS S, ALSHEHRI B, ALAIDAROUS M. Neurological Manifestation of SARSCoV-2 Induced Inflammation and Possible Therapeutic Strategies Against COVID19. Mol Neurobiol. 2021 Jul;58(7):3417-3434. doi: 10.1007/s12035-021-02318-9. Epub 2021 Mar 14. PMID: 33715108; PMCID: PMC7955900. Acesso: 13.09,2021.

LIU D, YANG J, FENG B, LU W, ZHAO C, LI L. Mendelian randomization analysis identified genes pleiotropically associated with the risk and prognosis of COVID-19. $\mathbf{J}$ Infect. 2021 Jan;82(1):126-132. doi: 10.1016/j.jinf.2020.11.031. Epub 2020 Nov 28. PMID: 33259846; PMCID: PMC7698677. Acesso: 13.09,2021.

PERRIN P, COLLONGUES N, BALOGLU S, BEDO D, BASSAND X, LAVAUX T, GAUTIER-VARGAS G, KELLER N, KREMER S, FAFI-KREMER S, MOULIN B, BENOTMANE I, CAILLARD S. Cytokine release syndrome-associated encephalopathy in patients with COVID-19. Eur J Neurol. 2021 Jan;28(1):248-258. doi: 10.1111/ene.14491. Epub 2020 Oct 5. PMID: 32853434; PMCID: PMC7461405. Acesso: 13.09,2021.

RANGANATHAN C, FUSINSKI SD, OBEID IM, ISMAIL KM, FERGUSON DT, RAMINICK MF, DAWES SM. Therapeutic plasma exchange for persistent encephalopathy associated with Covid-19. eNeurologicalSci. 2021 Mar;22:100327. 
doi: 10.1016/j.ensci.2021.100327. Epub 2021 Feb 7. PMID: 33585705; PMCID: PMC7868106. Acesso: 13.09,2021.

SAUSSEZ S, LECHIEN JR, HOPKINS C. Anosmia: an evolution of our understanding of its importance in COVID-19 and what questions remain to be answered. Eur Arch Otorhinolaryngol. 2021 Jul;278(7):2187-2191. doi: 10.1007/s00405-020-06285-0. Epub 2020 Sep 9. PMID: 32909060; PMCID: PMC7480210. Acesso: 13.09,2021.

SINGH H, SINGH A, KHAN AA, GUPTA V. Immune mediating molecules and pathogenesis of COVID-19-associated neurological disease. Microb Pathog. 2021 Sep;158:105023. doi: 10.1016/j.micpath.2021.105023. Epub 2021 Jun 4. PMID: 34090983; PMCID: PMC8177310. Acesso: 13.09,2021.

SOLOMON T. Neurological infection with SARS-CoV-2 - the story so far. Nat Rev Neurol. 2021 Feb;17(2):65-66. doi: 10.1038/s41582-020-00453-w. PMID: 33414554; PMCID: PMC7789883. Acesso: 13.09,2021.

SULLIVAN BN, FISCHER T. Age-Associated Neurological Complications of COVID19: A systematic Review and Meta-Analysis. Front Aging Neurosci. 2021 Aug 2;13:653694. doi: 10.3389/fnagi.2021.653694. PMID: 34408638; PMCID: PMC8366271. Acesso: 13.09,2021.

THEPMANKORN P, BACH J, LASFAR A, ZHAO X, SOUAYAH S, CHONG ZZ, SOUAYAH N. Cytokine storm induced by SARS-CoV-2 infection: The spectrum of its neurological manifestations. Cytokine. 2021 Feb;138:155404. doi: 10.1016/j.cyto.2020.155404. Epub 2020 Dec 11. PMID: 33360025; PMCID: PMC7832981. Acesso: 13.09,2021. 
EDUCAÇÃO, SAÚDE

ETECNÓLOGIA

26 A 28 DE OUTUBRO DE 2021

\section{QUFN}

XIA X, WANG Y, ZHENG J. COVID-19 and Alzheimer's disease: how one crisis worsens the other. TransI Neurodegener. 2021 Apr 30;10(1):15. doi:

10.1186/s40035-021-00237-2. PMID: 33941272; PMCID: PMC8090526. Acesso: $13.09,2021$. 